\title{
The electromotive force of MnAs nanoparticles
}

ARISING FRom P. N. Hai, S. Ohya, M. Tanaka, S. E. Barnes \& S. Maekawa. Nature 458, 489-492 (2009)

Magnetic tunnel junctions can produce large magnetoresistance effects that are of use in a variety of applications. Hai et al. ${ }^{1}$ recently published a very interesting paper in which the application of a large static magnetic field to a tunnel junction containing superparamagnetic MnAs nanoparticles resulted in the generation of an electromotive force. The authors attributed this phenomenon to a conversion of the nanoparticles' magnetic energy to electrical energy by way of quantum tunnelling. Here I point out that the electrical energy output measured by Hai et al. ${ }^{1}$ was more than 1,000 times greater than the maximum amount of magnetic energy that could be induced in their MnAs nanoparticles by the applied magnetic field. Therefore the induced magnetic energy cannot be the source for the observed electromotive force, as was asserted by Hai et al. ${ }^{1}$.

The maximum magnetic energy that can be induced in the MnAs nanoparticles by the applied magnetic field has the form $E_{\text {mag }}=2 N \mu B$, where $N$ is the number of nanoparticles in the sample, $\mu$ is the average saturation magnetic moment per nanoparticle, and $B$ is the applied magnetic field. The values of these parameters as determined by Hai et al. ${ }^{1}$ were $N \approx 10^{9}, B=10 \mathrm{kG}$ and $\mu=2 \mu_{\mathrm{B}} S$, where $S$ is the average spin per nanoparticle (in units of $\hbar$ ), $\sim 200$, and $\mu_{\mathrm{B}}$ is the Bohr magneton. This yields $E_{\mathrm{mag}} \approx 7 \times 10^{-12} \mathrm{~J}$ for their device. However, by using the inset of figure $2 \mathrm{c}$ in ref. 1 to calculate the electrical output power delivered to a $200 \mathrm{k} \Omega$ load resistor and integrating over time, it can be seen that the electrical energy output of the device was greater than $10^{-8} \mathrm{~J}$; this is more than a factor of 1,000 greater than $E_{\text {mag. }}$.

\section{C. Ralph ${ }^{1,2}$}

${ }^{1}$ Laboratory of Atomic and Solid State Physics, Cornell University, Ithaca, New York 14853, USA.

${ }^{2}$ Kavli Institute at Cornell, Cornell University, Ithaca, New York 14853, USA.

e-mail: ralph@ccmr.cornell.edu

Received 27 January; accepted 5 April 2011.

1. Hai, P. N., Ohya, S., Tanaka, M., Barnes, S. E. \& Maekawa, S. Electromotive force and huge magnetoresistance in magnetic tunnel junctions. Nature 458, 489-492 (2009).

Competing Interests: declared none.

doi:10.1038/nature10142 